\title{
GEOMETRY OF STAR-FORMING GALAXIES FROM SDSS, 3D-HST, AND CANDELS*
}

\author{
A. van der Wel ${ }^{1}$, Yu-Yen Chang ${ }^{1}$, E. F. Bell ${ }^{2}$, B. P. Holden $^{3}$, H. C. Ferguson ${ }^{4}$, M. Giavalisco ${ }^{5}$, H.-W. Rix ${ }^{1}$, R. Skelton $^{6}$, \\ K. Whitaker ${ }^{7}$, I. Momcheva ${ }^{8}$, G. Brammer ${ }^{4}$, S. A. Kassin ${ }^{4}$, M. Martig ${ }^{1}$, A. Dekel ${ }^{9}$, D. Ceverino ${ }^{10}$, D. C. Koo $^{3}$, \\ M. Mozena ${ }^{3}$, P. G. van DoKKum ${ }^{8}$, M. FranX $^{11}$, S. M. FABER ${ }^{3}$, AND J. PrimacK ${ }^{12}$ \\ ${ }^{1}$ Max-Planck Institut für Astronomie, Königstuhl 17, D-69117, Heidelberg, Germany; vdwel@ mpia.de \\ 2 Department of Astronomy, University of Michigan, 500 Church Street, Ann Arbor, MI 48109, USA \\ ${ }^{3}$ UCO/Lick Observatory, Department of Astronomy and Astrophysics, University of California, Santa Cruz, CA 95064, USA \\ ${ }^{4}$ Space Telescope Science Institute, 3700 San Martin Drive, Baltimore, MD 21218, USA \\ ${ }^{5}$ Astronomy Department, University of Massachusetts, Amherst, MA 01003, USA \\ ${ }^{6}$ South African Astronomical Observatory, P.O. Box 9, Observatory 7935, South Africa \\ ${ }^{7}$ Astrophysics Science Division, Goddard Space Center, Greenbelt, MD 20771, USA \\ ${ }^{8}$ Department of Astronomy, Yale University, New Haven, CT 06511, USA \\ ${ }^{9}$ Center for Astrophysics and Planetary Science, Racah Institute of Physics, The Hebrew University, Jerusalem 91904, Israel \\ ${ }^{10}$ Department of Theoretical Physics, Universidad Autonoma de Madrid, E-28049 Madrid, Spain \\ ${ }^{11}$ Leiden Observatory, Leiden University, P.O. Box 9513, NL-2300 AA Leiden, The Netherlands \\ ${ }^{12}$ Department of Physics, University of California at SantaCruz, Santa Cruz, CA 95064, USA \\ Received 2014 June 10; accepted 2014 July 15; published 2014 August 12
}

\begin{abstract}
We determine the intrinsic, three-dimensional shape distribution of star-forming galaxies at $0<z<2.5$, as inferred from their observed projected axis ratios. In the present-day universe, star-forming galaxies of all masses $10^{9}-10^{11} M_{\odot}$ are predominantly thin, nearly oblate disks, in line with previous studies. We now extend this to higher redshifts, and find that among massive galaxies $\left(M_{*}>10^{10} M_{\odot}\right)$ disks are the most common geometric shape at all $z \lesssim 2$. Lower-mass galaxies at $z>1$ possess a broad range of geometric shapes: the fraction of elongated (prolate) galaxies increases toward higher redshifts and lower masses. Galaxies with stellar mass $10^{9} M_{\odot}$ $\left(10^{10} M_{\odot}\right)$ are a mix of roughly equal numbers of elongated and disk galaxies at $z \sim 1(z \sim 2)$. This suggests that galaxies in this mass range do not yet have disks that are sustained over many orbital periods, implying that galaxies with present-day stellar mass comparable to that of the Milky Way typically first formed such sustained stellar disks at redshift $z \sim 1.5-2$. Combined with constraints on the evolution of the star formation rate density and the distribution of star formation over galaxies with different masses, our findings imply that, averaged over cosmic time, the majority of stars formed in disks.
\end{abstract}

Key words: galaxies: evolution - galaxies: fundamental parameters - galaxies: high-redshift - galaxies: structure

Online-only material: color figures

\section{INTRODUCTION}

The shape of the stellar body of a galaxy reflects its formation process. Reconstructing the intrinsic, three-dimensional (3D) shapes of spiral galaxies from their shapes projected on the sky has a long tradition, and proved to be an exquisitely accurate and precise approach, especially once sample size increased (e.g., Sandage et al. 1970; Lambas et al. 1992; Ryden 2004; Vincent \& Ryden 2005; Padilla \& Strauss 2008). These results provided us with the general notion that the stellar bodies of present-day star-forming galaxies over a wide range in luminosity can be described as thin, nearly oblate (therefore disk-like) systems with an intrinsic short-to-long axis ratio of $\sim 0.25$. Such global shapes encompass all galactic components, including bars and bulges. The disk component is generally thinner $(0.1-0.2$, e.g., Kregel et al. 2002).

Analogous information about the progenitors of today's galaxies is scarcer. Among faint, blue galaxies in deep Hubble Space Telescope (HST) imaging, Cowie et al. (1995) found a substantial population of elongated "chain" galaxies, but several authors argued that chain galaxies are edge-on disk galaxies (e.g., Dalcanton \& Shectman 1996; Elmegreen et al. 2004a,

\footnotetext{
* Based on observations with the Hubble Space Telescope, obtained at the Space Telescope Science Institute, which is operated by AURA, Inc., under NASA contract NAS 5-26555.
}

2004b). However, Ravindranath et al. (2006) demonstrated that the ellipticity distribution of a large sample of $z=2-4$ Lyman break galaxies is inconsistent with randomly oriented disk galaxies, lending credence to the interpretation that a class of intrinsically elongated (or, prolate) objects in fact exists at high redshift. By modeling ellipticity distributions, Law et al. (2012) and Yuma et al. (2012) concluded that their intrinsic shapes of $z>1.5$ star-forming galaxies are strongly triaxial.

On the other hand, regular rotation is commonly seen amongst $z \sim 1-2$ samples (Förster Schreiber et al. 2006, 2009; Kassin et al. 2007; Law et al. 2009; Wisnioski et al. 2011; Gnerucci et al. 2011; Newman et al. 2013), and the evidence for the existence of gaseous disks is ample among massive systems (Genzel et al. 2006; Wright et al. 2007; van Starkenburg et al. 2008; Stark et al. 2008; Epinat et al. 2009). One possible explanation for the seeming discrepancy between the geometric and kinematic shape inferences is a dependence of structure on galaxy mass. Indeed, for lower-mass galaxies $\left(\lesssim 10^{10} M_{\odot}\right)$ the evidence for rotation is less convincing (e.g., Förster Schreiber et al. 2006; Law et al. 2007) and in rare cases rotation is convincingly ruled out (e.g., Lowenthal et al. 2009). The prevailing view is that the gas-and hence presumably the stars that form from it - in those galaxies is supported by random motions rather than ordered rotation. However, the kinematic measurements for low-mass galaxies probe only a small number of spatial resolution elements - signs of rotation may be smeared out (Jones et al. 


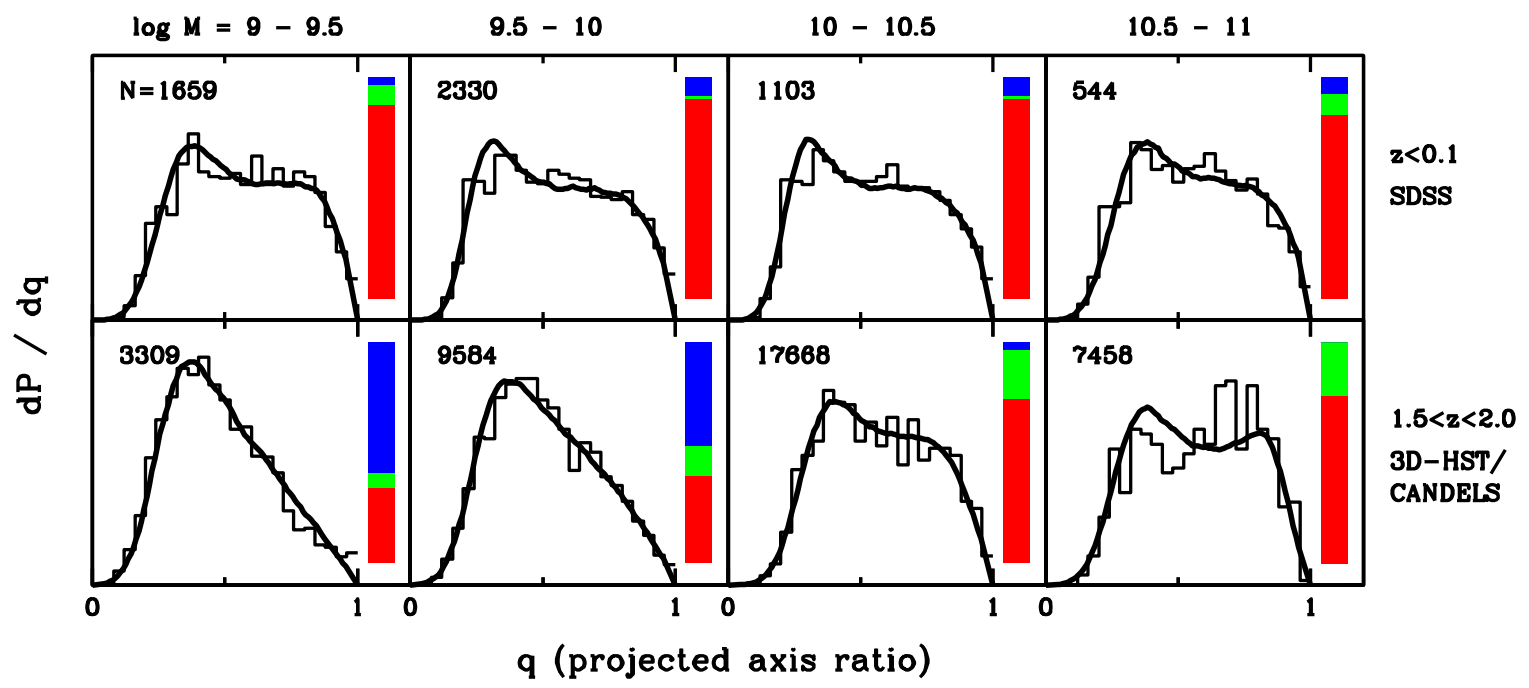

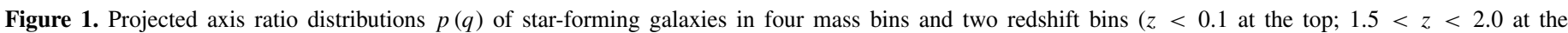

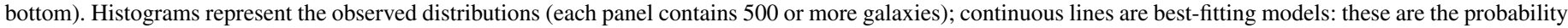

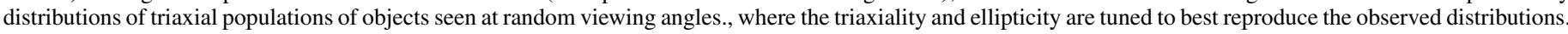

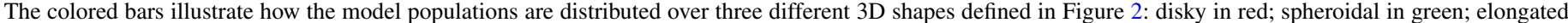
in blue. The pronounced variation among the projected axis ratio distributions illustrates that the changes in the geometric fractions are highly significant.

(A color version of this figure is available in the online journal.)

2010) — and the observed motions may have a non-gravitational origin such as feedback.

Here we aim to provide the first description of the geometric shape distribution of $z>1$ star-forming galaxies and its dependence on galaxy mass. We examine the projected axis ratio distributions $(p(q))$ of large samples of star-forming galaxies out to $z=2.5$ drawn from the CANDELS (Grogin et al. 2011; Koekemoer et al. 2011) and 3D-HST (Brammer et al. 2012; Skelton et al. 2014) surveys. A low-redshift comparison sample is drawn from the Sloan Digital Sky Survey (SDSS). The methodology developed by Holden et al. (2012) and Chang et al. (2013) will be used to convert $p(q)$ into 3D shape distributions of star-forming galaxies and its evolution from $z=2.5$ to the present day.

\section{DATA}

We construct volume-limited samples of star-forming galaxies over a large range in stellar mass $\left(10^{9}-10^{11} M_{\odot}\right)$ and redshift $(0<z<2.5)$ with $q$ measured at an approximately fixed restframe wavelength of $4600 \AA$.

\subsection{CANDELS and $3 D-H S T$}

Skelton et al. (2014) provide WFC3/F125W+F140W+ F160W-selected, multi-wavelength catalogs for the CANDELS fields, as well as redshifts, stellar masses, and rest-frame colors using the 3D-HST WFC3 grism spectroscopy in addition to the photometry. 36,653 star-forming galaxies with stellar masses $M_{*}>10^{9} M_{\odot}$ and up to redshift $z=2.5$ are selected based on their rest-frame $U-V$ and $V-J$ colors as described by van der Wel et al. (2014), 35,832 of which have $q$ measurements. The typical accuracy and precision is better than $10 \%$ (van der Wel et al. 2012). For the $2<z<2.5$ galaxies we use the F160Wbased values, for the $z<2$ galaxies we use the F125W-based values, such that all $z>1$ galaxies have their shapes measured at a rest-frame wavelength as close as possible to $4600 \AA$ (and always in the range $4300<\lambda / \AA<6200$ ). This avoids the effects due the shape variations with wavelength seen in local galaxies (Dalcanton \& Bernstein 2002).
Below $z=1$ our F125W shape measurements probe longer wavelengths. We compared the F125W-based shapes with HST/ Advanced Camera for Surveys F814W-based shapes for 1365 galaxies (see van der Wel et al. 2014). The median F125W-based axis ratio is 0.014 larger than the median F814W-based shape, with a scatter of 0.06. This is consistent with the measurement errors. We conclude that using F125W axis ratios at $z<1$ does not affect our results.

\subsection{SDSS: $0.04<z<0.08$}

SDSS photometry-based stellar masses from Brinchmann et al. (2004) are used to select 36,369 star-forming galaxies with stellar masses $M_{*}>10^{9} M_{\odot}$ and in the (spectroscopic) redshift range $0.04<z<0.08$. The distinction between star-forming and passive galaxies is described by Holden et al. (2012) and is based on the rest-frame $u-r$ and $r-z$ colors, analogous to the use of $U-V$ and $V-J$ colors at higher redshifts. For the SDSS sample we use the $q$ estimates from fitting the exponential surface brightness model to the $g$-band imaging as part of the DR7 photometric pipeline (Abazajian et al. 2009). These measurements have been verified by Holden et al. (2012), who showed that systematic offsets and scatter with respect to our GALFIT-based measurements are negligible.

\section{RECONSTRUCTION: FROM PROJECTED TO INTRINSIC SHAPES}

The very pronounced change of the projected shape distribution with redshift (Figure 1) immediately reveals that galaxy structure evolves with cosmic time. Especially at low stellar masses we see that a larger fraction of galaxies have flat projected shapes than at the present day. This observation underpins the analysis presented in the remainder of the Letter. Here we provide a brief description of the methodology to infer the intrinsic, 3D shapes of galaxies, outlined in detail by Chang et al. (2013).

We adopt the ellipsoid as the general geometric form to describe the shapes of galaxies. It has three, generally different, axis lengths ( $A \geqslant B \geqslant C)$, commonly used to define ellipticity 


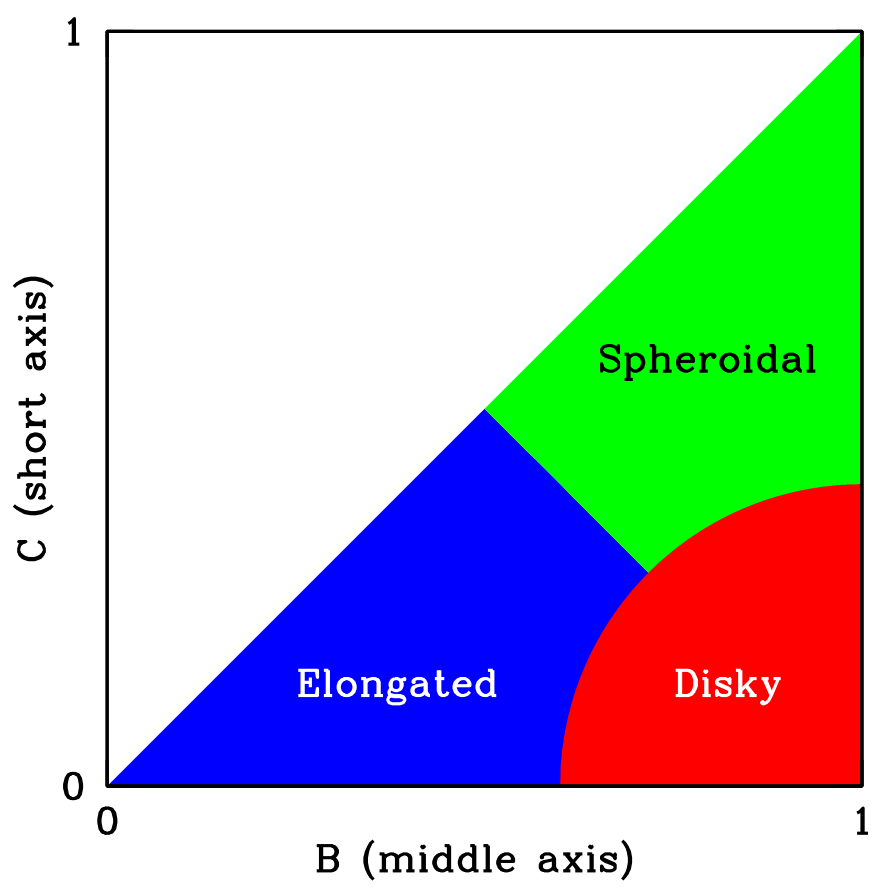

Figure 2. To facilitate a better intuitive understanding of the model shape parameters (triaxiality and ellipticity), we distinguish three crudely defined three-dimensional shapes of objects. Objects with three similarly long axes are defined as spheroidal; objects with two similarly long and one short axis are defined as disky; objects with one long axis and two similarly short axes are defined as elongated. A model population-generated to reproduce an observed axis ratio distribution - should be thought of as a cloud of points in the parameter space shown in this figure, distributed as prescribed by the best-fitting values of $T, \sigma_{T}, E$, and $\sigma_{E}$ (see the text for details). Each of the three regions will contain a given fraction of those points, that is, a fraction of the population.

(A color version of this figure is available in the online journal.)

$(1-C)$ and triaxiality $\left(\left(1-B^{2}\right) /\left(1-C^{2}\right)\right)$. In order to facilitate an intuitive understanding of our results, we define three broad geometric types, shown in Figure 2: disky $(A \sim B>C)$, elongated $(A>B \sim C)$, and spheroidal $(A \sim B \sim C)$.

The goal is to find a model population of triaxial ellipsoids that, when seen under random viewing angles, has the same $p(q)$ as an observed galaxy sample. Our model population has Gaussian distributions of the ellipticity (with mean $E$ and standard deviation $\sigma_{E}$ ) and triaxiality (with mean $T$ and standard deviation $\left.\sigma_{T}\right)$. Such a model population has a known $p(q)$ which we adjust to include the effect of random uncertainties in the axis ratio measurements - these are asymmetric for nearly round objects. Then, given that each observed value of $q$ corresponds to a known probability, we calculate the total likelihood of the model by multiplying the probabilities of each of the observed values. We search a grid of the four model parameters to find the maximal total likelihood.

In Figure 1 we show observed axis ratio distributions (histograms), and the probability distributions of the corresponding best-fitting model populations (smooth lines). The models generally match the data very well. Even in the worst case (bottom right panel) the model and data distributions are only marginally inconsistent, at the $2 \sigma$ level. A triaxial model population with parameters $\left(E, \sigma_{E}, T, \sigma_{T}\right)$ corresponds to a cloud of points in Figure 2 and, hence, with certain fractions of the three geometric types. The colored bars in Figure 1 represent these fractions for the best-fitting triaxial models. This illustrates the connection between projected shapes and intrinsic shapes: a broad $p(q)$ reflects a large fraction of disky objects, whereas a narrow distribution with a peak at small $q$ is indicative of a large fraction of elongated objects. A narrow distribution with a peak at large $q$ would indicate a large fraction of spheroidal objects.

In Figure 3 we provide the modeling results for the full redshift and mass range probed here: for each stellar mass bin we show the redshift evolution of the four model parameters, including the uncertainties obtained by bootstrapping the samples. Finally, in Figure 4 we show the full set of results in the form of the color coding defined in Figure 2.

\section{EVOLUTION OF INTRINSIC SHAPE DISTRIBUTIONS}

The small values of $T$ and the large values of $E$ for present-day star-forming galaxies (Figure 3 ) imply that the vast majority are thin and nearly oblate. Indeed, according to our classification shown in Figure 1 between $80 \%$ and $100 \%$ are disky, as is generally known and was demonstrated before on the basis of similar axis ratio distribution analyses by Vincent \& Ryden (2005) and Padilla \& Strauss (2008). Importantly, the intrinsic shape distribution of star-forming galaxies does not change over a large range in stellar mass $\left(10^{9}-10^{11} M_{\odot}\right)$.

Toward higher redshifts star-forming galaxies become gradually less disk-like (Figures 1, 3, and 4). This effect is most pronounced for low-mass galaxies. Already in the $0.5<z<1.0$ redshift bin in Figure 3 we see evolution, mostly in the scatter in triaxiality $\left(\sigma_{T}\right)$. That is, there is substantial variety in intrinsic galaxy shape. Beyond $z=1$, galaxies with stellar mass $10^{9} M_{\odot}$ typically do not have a disky geometry, but are most often elongated (Figure 3). Galaxies with mass $10^{10} M_{\odot}$ show similar behavior, but with evolution only apparent at $z>1.5$. This geometric evidence for mass-dependent redshift evolution of galaxy structure is corroborated by the analysis of kinematic properties of $z=0-1$ galaxies by Kassin et al. (2012).

Disky objects are the most common type $(\geqslant 75 \%)$ among galaxies with mass $>10^{10} M_{\odot}$ at all redshifts $z \lesssim 2$. A population of spheroidal galaxies is increasingly prominent among massive galaxies at $z>2$. A visual inspection of such objects reveals that at least a subset are mergers, but an in-depth interpretation of this aspect we defer to another occasion.

It is interesting to note that ellipticity hardly depends on mass and redshift (Figure 3). That is, despite strong evolution in geometry, the short-to-long axis ratio remains remarkably constant with redshift, and changes little with galaxy mass. A joint analysis of galaxy size and shape is required to explore the possible implications.

Note that our definition of geometric shape is unrelated to the common distinction between disks and spheroids on the basis of their concentration parameter or Sérsic index. As a result we distinguish between the observation that most low-mass starforming galaxies at $z \sim 2$ have exponential surface brightness profiles (e.g., Wuyts et al. 2011) and our inference that these galaxies are not, generally, shaped like disks in a geometric sense. This illustrates that an approximately exponential light profile can correlate with the presence of a disk-like structure but cannot be used as a definition of a disk.

\section{DISCUSSION}

Star formation in the present-day universe mostly takes place in $>10^{9} M_{\odot}$ galaxies and in non-starburst galaxies. Since essentially all such star-forming galaxies are disky and star formation in disk galaxies occurs mostly over the full extent of the stellar disk, it follows immediately that essentially all current star formation takes place in disks. The analysis presented in this 

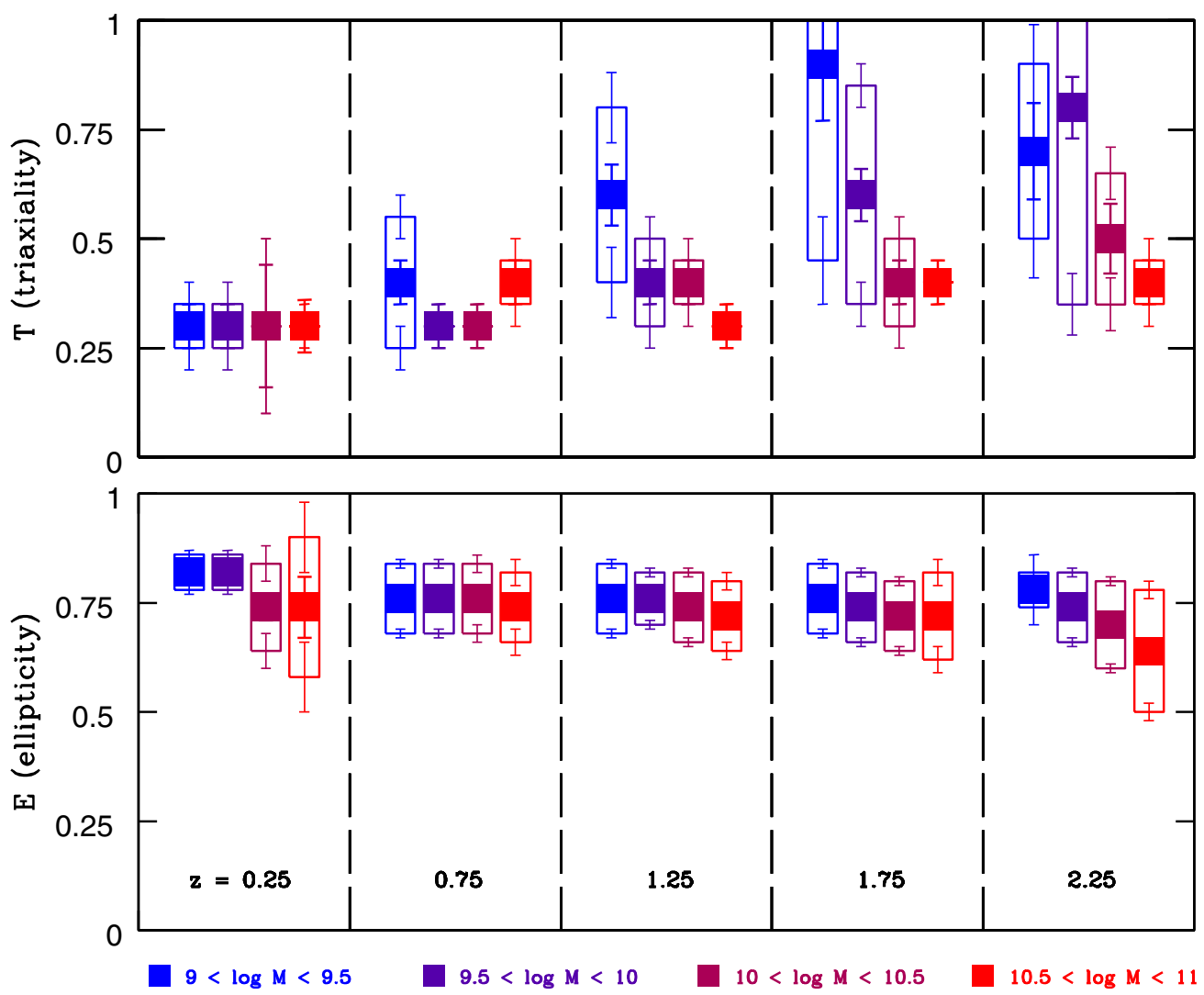

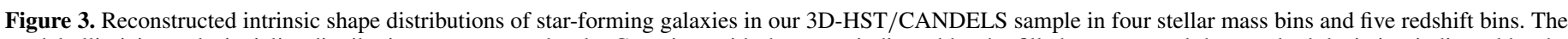

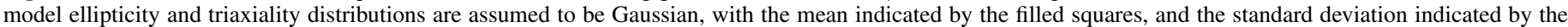

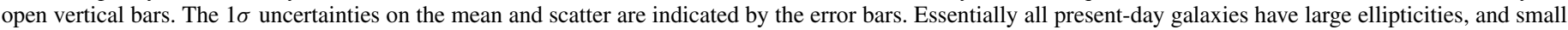

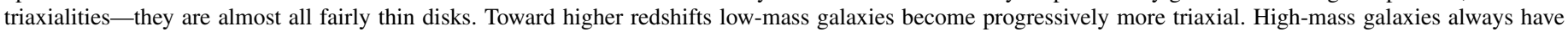
rather low triaxialities, but they become thicker at $z \sim 2$.

(A color version of this figure is available in the online journal.)

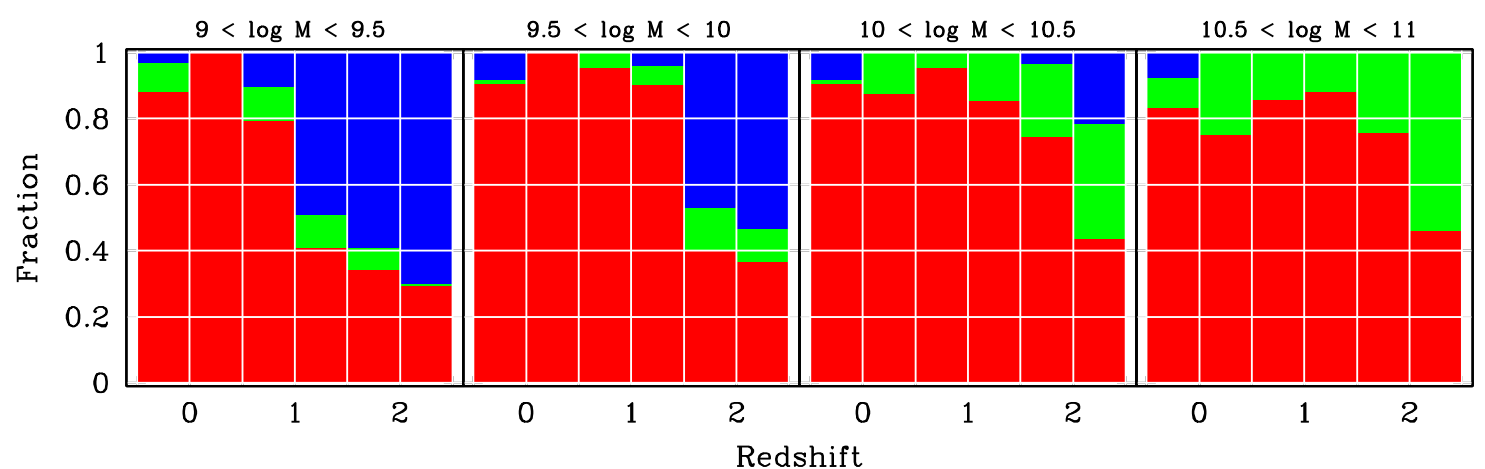

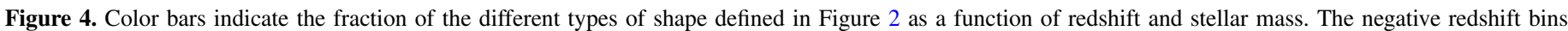
represent the SDSS results for $z<0.1$; the other bins are from 3D-HST/CANDELS.

(A color version of this figure is available in the online journal.)

Letter allows us to generalize this conclusion to include earlier epochs.

At least since $z \sim 2$ most star formation is accounted for by $\gtrsim 10^{10} M_{\odot}$ galaxies (e.g., Karim et al. 2011). Figures 3 and 4 show that such galaxies have disk-like geometries over the same redshift range. Given that $90 \%$ of stars in the universe formed over that time span, it follows that the majority of all stars in the universe formed in disk galaxies. Combined with the evidence that star formation is spatially extended, and not, for example, concentrated in galaxy centers (e.g., Nelson et al. 2012; Wuyts et al. 2012) this implies that the vast majority of stars formed in disks.
Despite this universal dominance of disks, the elongatedness of many low-mass galaxies at $z \gtrsim 1$ implies that the shape of a galaxy generally differs from that of a disk at early stages in its evolution. According to our results, an elongated, lowmass galaxy at $z \sim 1.5$ will evolve into a disk at later times, or, reversing the argument, disk galaxies in the present-day universe do not initially start out disks. ${ }^{13}$

As can be seen in Figure 3, the transition from elongated to disky is gradual for the population. This is not necessarily

13 This evolutionary path is potentially interrupted by the removal of gas and cessation of star formation. 
the case for individual galaxies. Hydrodynamical simulations indicate that sustained disks form quite suddenly, on a dynamical timescale, after an initial period characterized by rapidly changing dynamical configurations (e.g., Martig et al. 2014). This turbulent formation phase may include the subsequent formation and destruction of short-lived disks (e.g., Ceverino et al. 2014), associated with rapid changes in orientation and resulting in a hot stellar system of rather arbitrary shape.

Our observation that at $z>1$ the low-mass galaxy population consists of a mix of disky and elongated objects - in this picture, the latter represent the irregular phase without a sustained disk - can be interpreted as some fraction of the galaxies having already transformed into a sustained disk. The probability for this transition is, then, a function of mass which may or may not depend on redshift. Given the various estimates of the stellar mass evolution of Milky-Way-mass galaxies as a function of redshift (e.g., van Dokkum et al. 2013; Patel et al. 2013), we suggest that the Milky Way may have first attained a sustained stellar disk at redshift $z=1.5-2$.

\section{CAVEATS}

Our analysis rests on the assumption that stellar light traces the mass distribution of a galaxy. Potential spoilers include obscuration by dust, dispersion in age among stars, and large gas fractions.

Dust has a viewing angle-dependent effect on the measured $q$. Massive galaxies at all redshifts are dusty, and a large variety of dust geometries could disturb axis ratio measurements, hiding the disk-like structure of the population when traced by the axis ratio distribution. Perhaps this plays a role at $z>2$ where we see an increased fraction of round objects. However, the reverse- to create a disk-like axis ratio distribution for a population of dusty non-disks - requires unlikely fine tuning. We prefer the more straightforward interpretation that massive, star-forming galaxies truly are disks, at least up to $z=2$. This is supported by the observed correlation between axis ratio and color (e.g., Patel et al. 2012), also seen in our sample: galaxies with smaller $q$ are redder than those with larger $q$, as expected from a population on inclined, dusty disks.

Dust is also unlikely to affect $p(q)$ of low-mass galaxies. At $z>1$ galaxies with stellar masses $\lesssim 10^{10} M_{\odot}$ are generally very blue. For these young, presumably metal-poor galaxies dust is of limited relevance to the shape measurements. This also implies that completeness of our sample is not affected by strong dust obscuration.

Age variations in the stellar population and large gas fractions both potentially present challenges to our assumption that the rest-frame optical light traces the underlying mass distribution. Perhaps the luminous regions are young, bright complexes embedded in disks consisting of cold gas or fainter, older stellar populations. We cannot immediately discard this possibility as dynamical masses exceed stellar masses by an average factor of $\sim 3$ in the stellar mass range $10^{8} M_{\odot} \lesssim M_{*} \lesssim 10^{10} M_{\odot}$ galaxies at $z>1$ (e.g., Förster Schreiber et al. 2009; Maseda et al. 2013).

It is implausible that this difference between stellar mass and dynamical mass is entirely made up of undetected, older stars in a disk-like configuration. The different spatial distributions of the young and old stars would lead to wavelength-dependent shapes, which is not observed. If such a population of older stars is present, it must be spatially coincident with the young population, and not, generally, in a disk.

We cannot exclude the existence of cold gas disks that are $\sim 3$ times more massive than the (young) stellar population.
Hydrodynamical simulations show that low-mass, high-redshift systems can produce elongated stellar bodies embedded in more extended, turbulent gaseous bodies with ordered rotation (e.g., Ceverino et al. 2014). At the moment there is little observational evidence for such extended gaseous disks. For the mass range $10^{9.5} M_{\odot} \lesssim M_{*} \lesssim 10^{10} M_{\odot}$ gas masses in excess of the stellar mass have been inferred based on the star formation rate and the inverse Kennicutt-Schmidt relation (e.g., Förster Schreiber et al. 2009), but this inversion relies on the assumption of a disk-like geometry, weakening the argument. Furthermore, even if these cold gas mass estimates are correct it is not clear that the gas should be organized in a disk. Generally, gas ionized by star formation and cold gas share global kinematic traits, and in these cases the ionized gas does not generally show rotation. Deep ALMA observations will settle this issue, and for now we will leave this as the main caveat in our analysis.

\section{SUMMARY AND CONCLUSIONS}

We have analyzed the projected axis ratio distributions, $p(q)$, measured at rest-frame optical wavelengths, of stellar massselected samples of star-forming galaxies in the redshift range $0<z<2.5$ drawn from SDSS and 3D-HST+CANDELS. The intrinsic, 3D geometric shape distribution is reconstructed under the assumption that the population consists of triaxial objects view under random viewing angles.

In the present-day universe star-forming galaxies of all masses are predominantly oblate and flat, that is, they are disks. Massive galaxies $\left(M_{*}>10^{10} M_{\odot}\right)$ typically have this shape at all redshifts $0<z \lesssim 2$. Given the dominance of $10^{10}-10^{11} M_{\odot}$ galaxies in terms of their contribution to the cosmic stellar mass budget and the star formation rate density it follows that, averaged over all cosmic epochs, the majority of all stars formed in disks.

Lower-mass galaxies have shapes at $z>1$ that differ significantly from those of thin, oblate disks. For galaxies with stellar mass $10^{9} M_{\odot}\left(10^{10} M_{\odot}\right)$ there exists a mix of roughly equal numbers of elongated and disk galaxies at $z \sim 1(z \sim 2)$. At $z>1$ the $10^{9} M_{\odot}$ galaxies are predominantly elongated. Our findings imply that low-mass galaxies at high redshift had not yet formed a regularly rotating, sustained disk. Given a range of plausible mass growth rate of Milky Way-mass galaxies we infer the disk formation phase for such galaxies at $z=1.5-2$.

\section{REFERENCES}

Abazajian, K. N., Adelman-McCarthy, J. K., Agüeros, M. A., et al. 2009, ApJS, 182,543

Brammer, G. B., van Dokkum, P. G., Franx, M., et al. 2012, ApJS, 200, 13 Brinchmann, J., Charlot, S., White, S. D. M., et al. 2004, MNRAS, 351, 1151 Ceverino, D., Klypin, A., Klimek, E., et al. 2014, MNRAS, 442, 1545 Chang, Y.-Y., van der Wel, A., Rix, H.-W., et al. 2013, ApJ, 773, 149

Cowie, L. L., Hu, E. M., \& Songaila, A. 1995, AJ, 110, 1576

Dalcanton, J. J., \& Bernstein, R. A. 2002, AJ, 124, 1328

Dalcanton, J. J., \& Shectman, S. A. 1996, ApJL, 465, L9

Elmegreen, D. M., Elmegreen, B. G., \& Hirst, A. C. 2004a, ApJL, 604, L21 Elmegreen, D. M., Elmegreen, B. G., \& Sheets, C. M. 2004b, ApJ, 603, 74 Epinat, B., Contini, T., Le Fèvre, O., et al. 2009, A\&A, 504, 789

Förster Schreiber, N. M., Genzel, R., Bouché, N., et al. 2009, ApJ, 706, 1364

Förster Schreiber, N. M., Genzel, R., Lehnert, M. D., et al. 2006, ApJ, 645,1062

Genzel, R., Tacconi, L. J., Eisenhauer, F., et al. 2006, Natur, 442, 786

Gnerucci, A., Marconi, A., Cresci, G., et al. 2011, A\&A, 528, A88

Grogin, N. A., Kocevski, D. D., Faber, S. M., et al. 2011, ApJS, 197, 35

Holden, B. P., van der Wel, A., Rix, H.-W., \& Franx, M. 2012, ApJ, 749, 96

Jones, T. A., Swinbank, A. M., Ellis, R. S., Richard, J., \& Stark, D. P. 2010, MNRAS, 404, 1247

Karim, A., Schinnerer, E., Martnez-Sansigre, A., et al. 2011, ApJ, 730, 61 
Kassin, S. A., Weiner, B. J., Faber, S. M., et al. 2007, ApJL, 660, L35 Kassin, S. A., Weiner, B. J., Faber, S. M., et al. 2012, ApJ, 758, 106 Koekemoer, A. M., Faber, S. M., Ferguson, H. C., et al. 2011, ApJS, 197, 36

Kregel, M., van der Kruit, P. C., \& de Grijs, R. 2002, MNRAS, 334, 646 Lambas, D. G., Maddox, S. J., \& Loveday, J. 1992, MNRAS, 258, 404

Law, D. R., Steidel, C. C., Erb, D. K., et al. 2007, ApJ, 669, 929

Law, D. R., Steidel, C. C., Erb, D. K., et al. 2009, ApJ, 697, 2057

Law, D. R., Steidel, C. C., Shapley, A. E., et al. 2012, ApJ, 745, 85

Lowenthal, J. D., Koo, D. C., Simard, L., \& van Kampen, E. 2009, ApJ, 703, 198

Martig, M., Minchev, I., \& Flynn, C. 2014, arXiv:1405.1727

Maseda, M. V., van der Wel, A., da Cunha, E., et al. 2013, ApJL, 778, L22

Nelson, E. J., van Dokkum, P. G., Brammer, G., et al. 2012, ApJL, 747, L28

Newman, S. F., Genzel, R., Förster Schreiber, N. M., et al. 2013, ApJ, 767, 104

Padilla, N. D., \& Strauss, M. A. 2008, MNRAS, 388, 1321

Patel, S. G., Fumagalli, M., Franx, M., et al. 2013, ApJ, 778, 115
Patel, S. G., Holden, B. P., Kelson, D. D., et al. 2012, ApJL, 748, L27

Ravindranath, S., Giavalisco, M., Ferguson, H. C., et al. 2006, ApJ, 652, 963 Ryden, B. S. 2004, ApJ, 601, 214

Sandage, A., Freeman, K. C., \& Stokes, N. R. 1970, ApJ, 160, 831

Skelton, R. E., Whitaker, K. E., Momcheva, I. G., et al. 2014, arXiv:1403.3689

Stark, D. P., Swinbank, A. M., Ellis, R. S., et al. 2008, Natur, 455, 775

van der Wel, A., Bell, E. F., Häussler, B., et al. 2012, ApJS, 203, 24

van der Wel, A., Franx, M., van Dokkum, P. G., et al. 2014, ApJ, 788, 28

van Dokkum, P. G., Leja, J., Nelson, E. J., et al. 2013, ApJL, 771, L35

van Starkenburg, L., van der Werf, P. P., Franx, M., et al. 2008, A\&A, 488, 99

Vincent, R. A., \& Ryden, B. S. 2005, ApJ, 623, 137

Wisnioski, E., Glazebrook, K., Blake, C., et al. 2011, MNRAS, 417, 2601

Wright, S. A., Larkin, J. E., Barczys, M., et al. 2007, ApJ, 658, 78

Wuyts, S., Förster Schreiber, N. M., Genzel, R., et al. 2012, ApJ, 753, 114

Wuyts, S., Förster Schreiber, N. M., van der Wel, A., et al. 2011, ApJ, 742, 96

Yuma, S., Ohta, K., \& Yabe, K. 2012, ApJ, 761, 19 